Rev. Inst. Flor. v. 26 n. 1 p. 7-15 2014

http://dx.doi.org/10.4322/rif.2014.001

ISSN impresso 0103-2674/on-line 2178-5031

\title{
RETENCIÓN DE CARBONO EN RODALES PARA LA PRODUCCIÓN DE MADERA EN Pinus caribaea Morelet var. caribaea B. \& G., EN LA REGIÓN DE TOPE DE COLLANTES, GUAMUHAYA, PROVINCIA SANCTI SPIRITUS ${ }^{1}$
}

\section{CARBON RETENTION IN FOREST STANDS IN Pinus caribaea Morelet var. caribaea B. \& G. FOR WOOD PRODUCTION IN THE REGION OF TOPE DE COLLANTES, GUAMUHAYA, SANCTI SPIRITUS PROVINCE}

\author{
Alicia MERCADET ${ }^{2,3}$; Arnaldo ÁLVAREZ2; Yolanis RODRÍGUEZ²; \\ Luis M. GÓMEZ²; Manuel VALLE²; Roberto RAMOS; \\ Dariel MORALES'; Jesús TOLEDO²; Jorge LEÓN ${ }^{2}$
}

\begin{abstract}
RESUMEN - En Cuba no existen antecedentes sobre cómo abordar de forma integrada la producción de madera para aserrío y la retención de carbono. Se establecieron 30 parcelas temporales de $500 \mathrm{~m}^{2}$ en plantaciones de Pinus caribaea Morelet var. caribaea Barret y Golfari en la región de Tope de Collantes, ubicada en el macizo montañoso de Guamuhaya, provincia Sancti Spiritus, Cuba; en ellas se midieron el diámetro normal $\left(\mathrm{d}_{1,30}\right)$, la altura total y el grosor de corteza por árbol (GCa), calculando el volumen total con corteza por árbol (VTcca), el rendimiento por hectárea $(\mathrm{R})$ y la retención de carbono $(\mathrm{C})$. Se analizaron las tendencias de variación de estas variables con el espaciamiento manteniendo fijos los efectos sitio y edad primero y confundiendo el efecto sitio dentro del efecto espaciamiento después. En todos los casos el $\mathrm{d}_{1,30}$ y el VTcca presentaron tendencias ascendentes con el aumento del espaciamiento, en tanto que el $\mathrm{GCa}$, el $\mathrm{R}$ y el $\mathrm{C}$ presentaron tendencias descendentes, sugiriendo que para combinar la producción de madera para aserrío con la retención de carbono, resulta conveniente identificar un espaciamiento de compromiso entre ambas variables, que para esta especie y en estas condiciones resultó ser de 990 árboles*ha ${ }^{-1}$, equivalente a 3,2 m x 3,2 m.
\end{abstract}

Palabras clave: carbono; madera; espaciamiento; Pinus caribaea.

\begin{abstract}
In Cuba there are no precedents about how to manage wood plantations for sawmill, together with carbon retention. There were used 30 temporal plots $\left(500 \mathrm{~m}^{2}\right.$ each) in plantations of Pinus caribaea M. var. caribaea B. \& G. in Tope de Collantes, region of Cuban south-centre mountains in Sancti Spiritus province. In each plot were measured normal diameter $\left(\mathrm{d}_{1,30}\right)$, total height and bark thickness by tree, and then it was calculated total volume with bark per tree, yield per hectare and carbon retention in order to analyse variation tendencies of those variables with spacing, using first as fixed effects site and age and then, confounding site effect within spacing. In all cases normal diameter and total volume with bark per tree had increasing tendencies with respect to spacing, while bark thickness by tree, yield per hectare and carbon retention presented decreasing ones. Those results suggest that for an adequate combination of wood production for sawmill together with carbon retention, it would be convenient to establish a compromise spacing between both variables, which for that species and site conditions was 990 trees per hectare, equivalent to $3,2 \mathrm{~m} \times 3,2 \mathrm{~m}$.
\end{abstract}

Keywords: carbon; wood; spacing; Pinus caribaea.

${ }^{1}$ Recebido para análise em 14.08.13. Aceito para publicação em 03.04.14.

${ }^{2}$ Instituto de Investigaciones Agro-Forestales, Calle 174 No 1723 e/ 17B y 17C, Siboney, Playa, La Habana, Cuba.

${ }^{3}$ Autor para correspondência: Alicia Mercadet - mercadet@forestales.co.cu 


\section{INTRODUCCIÓN}

La determinación adecuada de la biomasa de un bosque, es un elemento de gran importancia debido a que ésta permite determinar los montos de carbono por unidad de superficie y tipo de bosques, además de otros elementos químicos existentes en cada uno de sus componentes (Snowdon et al., 2001; Montero et al., 2004). Esta información es de especial importancia en la actualidad, debido a la necesidad de conocer los montos de carbono capturado por los bosques naturales y plantaciones. La capacidad de los ecosistemas forestales para almacenar carbono en forma de biomasa aérea varía en función de la composición florística, la edad y la densidad de población de cada estrato por comunidad vegetal (Rodríguez et al., 2009).

Habitualmente, los rodales productores de madera para aserrío son manejados silviculturalmente buscando la optimización del volumen por árbol, de manera tal que una vez realizado el aprovechamiento, el bolo resultante presente características que favorezcan la máxima eficiencia del procesamiento industrial, entre las que se incluyen un diámetro y longitud tan grandes como sea posible y una diferencia lo menor posible entre los diámetros de sus extremos, a lo que frecuentemente se adicionan otros aspectos relacionados con la calidad del producto industrial (cantidad y tamaño de los nudos, ausencia de áreas de crecimiento tensionado, etc.). Por estas razones es que en el manejo silvícola de estos rodales el raleo desempeña un papel principal (Burley, et al., 2004; Bravo y Montalvo, 2011).

Sin embargo, cuando los objetivos perseguidos con el rodal se modifican y a la producción de madera para aserrío se adiciona como interés la retención de carbono, se añade a los intereses anteriores la maximización de la cantidad de biomasa por unidad de superficie y con ello, de la cantidad de carbono retenido.

Pero la maximización del volumen por árbol mediante la aplicación de raleos (aclareos) sucesivos implica, de hecho, la reducción de la cantidad total de biomasa por unidad de superficie y a su vez, del carbono total retenido, lo que genera un conflicto entre objetivos e indica la conveniencia de establecer un punto de compromiso entre ambas variables, que permita alcanzar su optimización, objetivo que se aborda con el presente trabajo en plantaciones de Pinus caribaea Morelet var. caribaea Barret y Golfari, en la zona de Tope de Collantes, Macizo de Guamuhaya, provincia Sancti Spiritus, Cuba.

\section{MATERIALES Y MÉTODOS}

El trabajo fue realizado en las zonas conocidas como: (1) La Chispa, (2) La Felicidad, (3) Los Puriales y (4) Tres Palmas, ubicadas todas en Tope de Collantes, en el Macizo de Guamuhaya, sobre suelo Ferralítico Rojo Lixiviado (Rubio, 1986), con un clima caracterizado por una temperatura mínima media mensual inferior a $20{ }^{\circ} \mathrm{C}$; temperatura máxima media mensual de $32{ }^{\circ} \mathrm{C}$; pluviosidad anual superior a los $2.000 \mathrm{~mm}$ y humedad relativa media anual de $80 \%$ (Hechavarría, 2009). En estos suelos la arcilla puede llegar al 63\%; no obstante, presentan un buen drenaje, son de reacción ácida, bajos en materia orgánica y pobres en casi todos los nutrientes pero, a causa de la altitud del lugar, las altas precipitaciones y la notable profundidad del suelo, el crecimiento de los árboles es de los mejores en Cuba (Bari et al., 1973).

En estas condiciones fueron establecidas un total de 30 parcelas temporales de muestreo de $500 \mathrm{~m}^{2}$ de superficie $(20 \mathrm{~m} \times 25 \mathrm{~m})$ en plantaciones productoras de madera para aserrío de Pinus caribaea M. var. caribaea B. \& G.; de ellas siete de 35 años en La Felicidad y el resto de 38 años, con una densidad que varió entre 500 y 1.520 árboles* ha $^{-1}$ (Tabla 1; Figura 1 ) y en cada parcela se midió el diámetro normal $\left(\mathrm{d}_{1,30}\right)$ con forcípula $( \pm 0,5 \mathrm{~cm})$ o con cinta diamétrica $( \pm 0,1 \mathrm{~cm})$, la altura total con hipsómetro Blume-Leis $( \pm 0,1 \mathrm{~m})$ y el grosor de corteza con medidor de corteza $( \pm 0,5 \mathrm{~mm})$ a todos los árboles de la parcela.

Tabla 1. Características de las parcelas temporales de muestreo establecidas.

Table 1. Characteristics of temporary sampling plots established.

\begin{tabular}{c|cccc}
\hline Lugar & Lote & Rodal & $\begin{array}{c}\text { Cant. } \\
\text { Parc. }\end{array}$ & $\begin{array}{c}\text { Densidad } \\
\text { (árb/ha) }\end{array}$ \\
\hline \multirow{2}{*}{ La Chispa* $^{*}$} & 5 & 2 & 3 & $1040-1100$ \\
& & 3 & 6 & $760-960$ \\
\hline \multirow{3}{*}{ La Felicidad** } & 8 & 2 & 2 & $960-1060$ \\
\cline { 2 - 5 } & 9 & 2 & 3 & $1120-1520$ \\
\hline \multirow{2}{*}{ Los Puriales* } & 8 & 5 & 2 & $500-540$ \\
\hline \multirow{3}{*}{ Tres Palmas* } & & 1 & 2 & $640-1500$ \\
& \multirow{2}{*}{6} & 2 & 1 & $740-1500$ \\
\cline { 2 - 5 } & & 4 & 1 & 1200 \\
& 7 & 3 & 2 & $960-820$ \\
& & 4 & 1 & 903 \\
\hline
\end{tabular}

*Parcelas de 38 años; **Parcelas de 35 años.

*Plots of 38 years old; **Plots of 35 years old. 
MERCADET, A. et al. Retención de carbono en rodales de Pinus caribaea Morelet var. caribaea B. \& G. en Tope de Collants

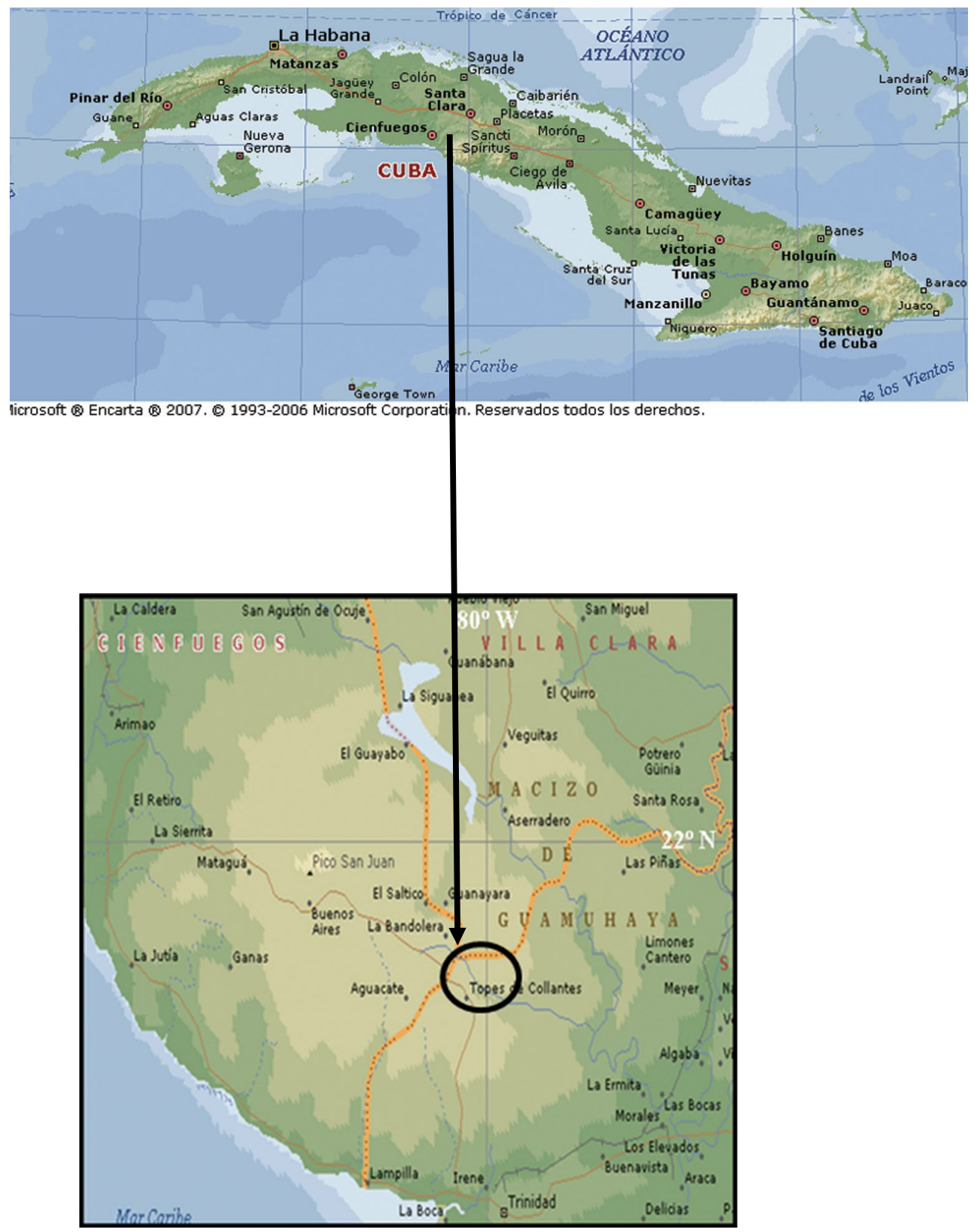

Figura 1. Ubicación del área de trabajo.

Figure 1. Workspace location. 

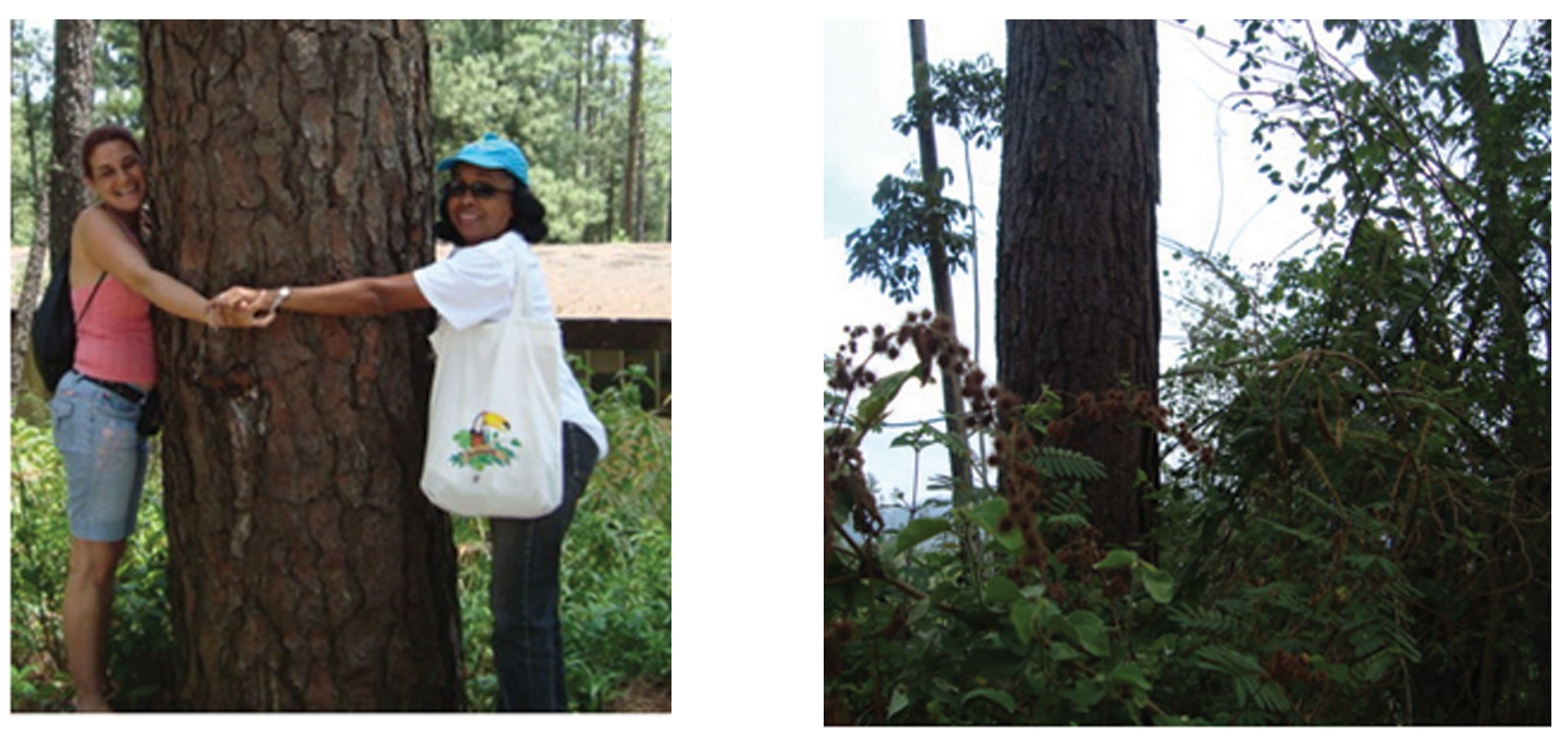

Figura 2. Ejemplo de árboles presentes en Tope de Collantes, provincia Sancti Spiritus.

Figure 2. Example of trees present in Tope de Collantes, Sancti Spiritus province.

El volumen total con corteza por árbol (VTcca) fue calculado según la expresión:

$$
\mathrm{VTcca}=\pi \mathrm{D} 2(\mathrm{H}+3) \mathrm{C}_{\mathrm{m}}
$$

donde:

$\pi$ - constante $(3,14159)$

$\mathrm{D}$ - diámetro a $1,30 \mathrm{~m}$ con corteza

$\mathrm{Cm}$ - coeficiente mórfico $(0,47)$

$\mathrm{H}$ - altura total

El rendimiento $\left(\mathrm{R} ; \mathrm{m}^{3 *} \mathrm{ha}^{-1}\right)$ se obtuvo como el producto del volumen medio de los árboles de la parcela, por la cantidad de árboles por hectárea que contenía; la biomasa seca del fuste de cada árbol $\left(\mathrm{t}^{*} \mathrm{ha}^{-1}\right)$ fue calculada como el producto del VTcca por la densidad básica promedio de la madera $\left(495 \mathrm{~kg}^{*} \mathrm{~m}^{-3}\right)$, obtenida a partir de los reportes de Carreras y Dechamps (1995), Soler (2001) e International Panel on Climate Change - IPCC (2006) y el contenido de carbono del fuste fue calculado como el producto de la biomasa seca por el coeficientede carbono para la madera (0,4753; Mercadet et al., 2011). 
Manteniendo constante el sitio y la edad pero variando la cantidad de árboles por hectárea (como expresión del espaciamiento), se estimó la tendencia de variación expresada por los valores medios por parcela para: (1) el $\mathrm{d}_{1,30}$, (2) el grosor de corteza, (3) el volumen por árbol, (4) el rendimiento y (5) el contenido de carbono, asumiendo un modelo lineal.

Posteriormente, manteniendo constante solo la edad, se confundieron los datos de las parcelas de igual edad (38 años) de todos los sitios en una sola muestra y se repitió el análisis anterior para cada variable.

Finalmente, para definir la cantidad de árboles/ha y el espaciamiento medio que en las condiciones del macizo de Guamuhaya, permitiera optimizar tanto el volumen por árbol, como la cantidad de biomasa seca de fuste por hectárea y con ello, la retención de carbono por unidad de superficie, se procedió a establecer la igualdad entre los modelos lineales:

$$
\mathrm{VTcc}=\mathrm{a}+\mathrm{b}\left(\text { Árb.ha }^{-1}\right) \text { [2] y R }=\mathrm{a}+\mathrm{b}\left(\text { Árb.ha }^{-1}\right) \text { [3] }
$$

\section{RESULTADOS Y DISCUSIÓN}

Al mantener constantes el sitio y la edad, tanto el $\mathrm{d}_{1,30}$ como el volumen por árbol presentaron tendencias ascendentes con la disminución de la cantidad de árboles por hectárea (aumento del espaciamiento), mientras que el grosor de corteza, el rendimiento y el carbono por hectárea presentaron tendencias descendentes, en los cuatro sitios considerados (Tabla 2).

Tabla 2. Resultados de las tendencias con respecto a la disminución de la cantidad de árboles por hectárea (variable independiente - X), por lugares y variables.

Table 2. Results of trends with respect to decreasing the number of trees per hectare (independent variable - X), by locations and variables.

\begin{tabular}{|c|c|c|c|c|}
\hline Lugar & $\begin{array}{l}\text { Variable Dependiente } \\
\text { (Y) }\end{array}$ & $\begin{array}{c}\text { Constante } \\
\text { Regresión } \\
\text { (a) }\end{array}$ & $\begin{array}{l}\text { Coeficiente } \\
\text { Regresión } \\
\text { (b) }\end{array}$ & $\begin{array}{c}\text { Coeficiente } \\
\text { Determinación } \\
\left(\mathrm{R}^{2}\right)\end{array}$ \\
\hline \multirow{5}{*}{ Los Puriales } & Diámetro normal $(\mathrm{cm})$ & 38,1520 & $-0,0038$ & 0,0478 \\
\hline & Corteza $(\mathrm{mm})$ & 0,5795 & 0,0008 & 0,2718 \\
\hline & Vol. por árbol (m³) & 1,4455 & $-0,0001$ & 0,0052 \\
\hline & Rendimiento $\left(\mathrm{Mm}^{3} / \mathrm{ha}\right)$ & 0,1078 & 0,0012 & 0,2608 \\
\hline & $\mathrm{C}$ en fuste (t/ha) & 25,3680 & 0,2855 & 0,2608 \\
\hline \multirow{5}{*}{ La Felicidad } & Diámetro normal $(\mathrm{cm})$ & 42,6610 & $-0,0053$ & 0,2975 \\
\hline & Corteza $(\mathrm{mm})$ & 1,0016 & 0,0003 & 0,4900 \\
\hline & Vol. por árbol $\left(\mathrm{m}^{3}\right)$ & 1,7279 & $-0,0003$ & 0,1024 \\
\hline & Rendimiento $\left(\mathrm{Mm}^{3} / \mathrm{ha}\right)$ & 0,3178 & 0,0011 & 0,7167 \\
\hline & $\mathrm{C}$ en fuste (t/ha) & 176,8900 & 0,2459 & 0,1197 \\
\hline \multirow{5}{*}{ Tres Palmas } & Diámetro normal $(\mathrm{cm})$ & 39,6470 & $-0,0048$ & 0,1473 \\
\hline & Corteza (mm) & 0,7901 & 0,0009 & 0,6095 \\
\hline & Vol. por árbol (m³) & 1,7134 & $-0,0003$ & 0,0657 \\
\hline & Rendimiento $\left(\mathrm{Mm}^{3} / \mathrm{ha}\right)$ & 0,2526 & 0,0012 & 0,7151 \\
\hline & $\mathrm{C}$ en fuste (t/ha) & 59,3770 & 0,2779 & 0,7137 \\
\hline \multirow{5}{*}{ La Chispa } & Diámetro normal $(\mathrm{cm})$ & 50,0590 & $-0,0152$ & 0,1622 \\
\hline & Corteza $(\mathrm{mm})$ & 0,6493 & 0,0011 & 0,2235 \\
\hline & Vol. por árbol $\left(\mathrm{m}^{3}\right)$ & 2,0853 & $-0,0009$ & 0,0772 \\
\hline & Rendimiento $\left(\mathrm{Mm}^{3} / \mathrm{ha}\right)$ & 0,6772 & 0,0005 & 0,0369 \\
\hline & $\mathrm{C}$ en fuste $(\mathrm{t} / \mathrm{ha})$ & 159,3200 & 0,1231 & 0,0369 \\
\hline
\end{tabular}


Estos resultados sugieren que, en la medida que aumenta el espaciamiento como resultado de los raleos (aclareos), el árbol tiende a presentar un mayor diámetro, un mayor volumen total con corteza, una corteza más fina y una menor cantidad de biomasa y de carbono, ambos por unidad de superficie, lo cual presentó similares comportamientos en los cuatro sitios evaluados, a edades constantes en cada uno de ellos.
Cuando el efecto sitio fue confundido dentro del efecto espaciamiento, dejando como efecto fijo solo la edad (en todos los casos, 38 años), los resultados presentaron las mismas tendencias antes explicadas (Figura 3), sugiriendo que tales comportamientos están más influenciados por la cantidad de árboles por hectárea, que por las diferencias entre lugares.
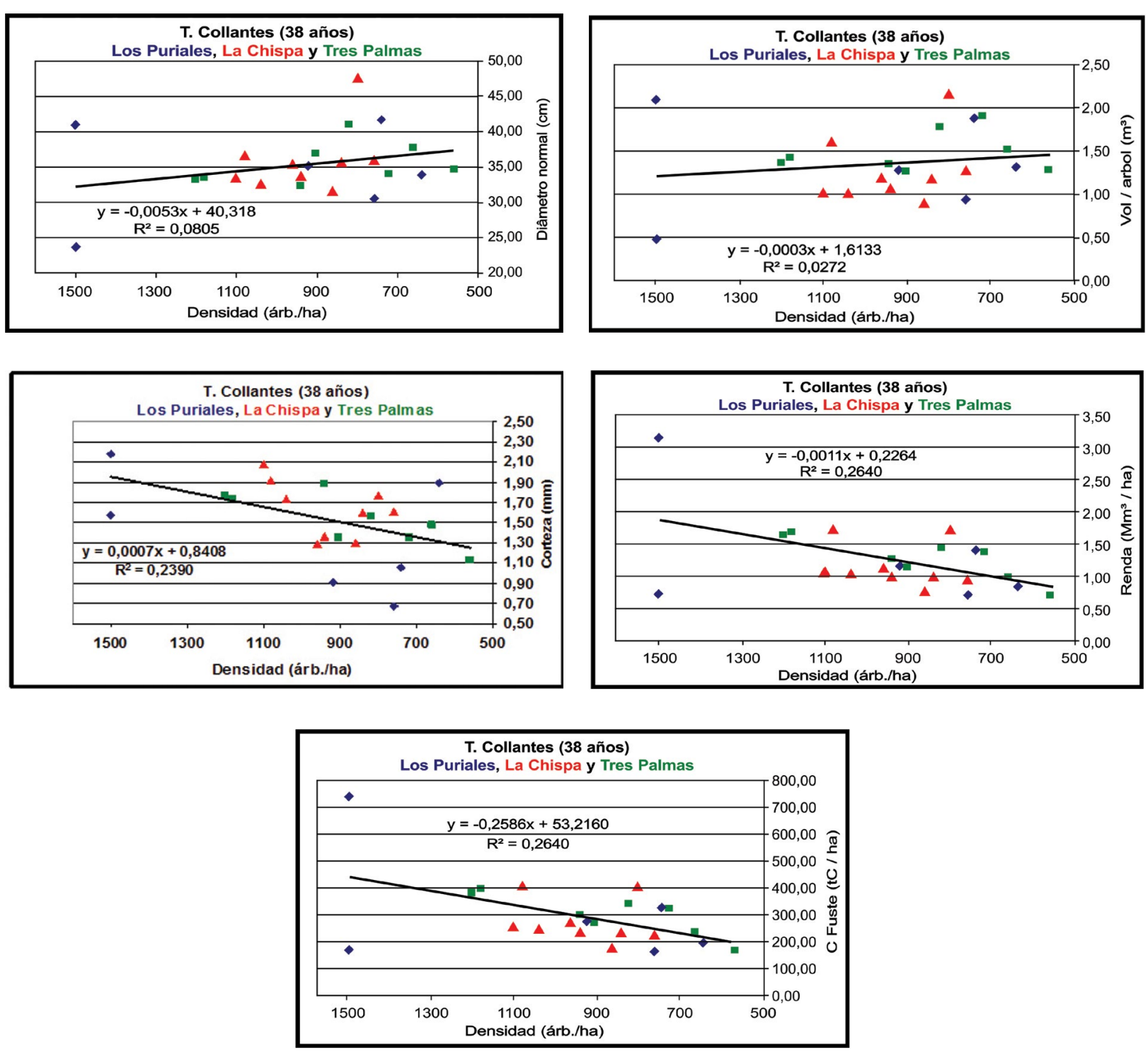

Figura 3. Comportamiento de las tendencias de variación ascendentes $\left(\mathrm{d}_{1,30} \mathrm{y}\right.$ volumen por árbol) y descendentes (grosor de corteza, rendimiento y carbono por hectárea) al confundir el efecto sitio en el espaciamiento. Leyenda: Los Puriales: $\approx$ La Chispa: $\triangle$ Tres Palmas: $\triangleright$.

Figure 3. Behavior trends ascendant variation $\left(\mathrm{d}_{1,30}\right.$ and volume per tree) and descending (bark thickness, yield and carbon per hectare) mixing the site effect with the spacing. Legend: Los Puriales: $₫$ La Chispa: $\Delta$ Tres Palmas: $\diamond$. 
MERCADET, A. et al. Retención de carbono en rodales de Pinus caribaea Morelet var. caribaea B. \& G. en Tope de Collants

En particular, el comportamiento del grosor de corteza registrado indica por una parte, que su importancia relativa en el volumen total con corteza disminuye con el aumento del espaciamiento y que la relación "volumen de madera/volumen de corteza" del árbol tiende a aumentar en magnitud, en la medida en que la cantidad de árboles por hectárea disminuye. Si a estos elementos se añade lo reportado por Mercadet et al. (2011), señalando la existencia de importantes diferencias del contenido de carbono en la madera y la corteza de esta especie, entonces resultaría conveniente en un trabajo posterior profundizar sobre estos aspectos.
La identificación del espaciamiento que permitiera maximizar tanto el volumen total por árbol para la producción de madera aserrada, como el rendimiento (estimado en este caso del total de biomasa y del carbono por unidad de superficie), alcanzó resultados muy similares tanto en cada uno de los sitios evaluados (fijando los efectos sitio y edad) (Tabla 3), como en el caso en que el efecto sitio fue confundido con el efecto cantidad de árboles por hectárea, variando entre un máximo de 1.029 árb.ha en Los Puriales y un mínimo de 974 árb./ha en Tres Palmas, equivalentes a un espaciamiento que oscilaría entre $3,12 \mathrm{~m} \mathrm{x} \mathrm{3,12} \mathrm{m}\left(9,72 \mathrm{~m}^{2}\right)$ y $3,20 \mathrm{~m} \times 3,20 \mathrm{~m}\left(10,27 \mathrm{~m}^{2}\right)$ (Figura 4).

Tabla 3. Estimación del espaciamiento $(\mathrm{CAH})$ de optimización para la combinación de la producción de madera para aserrío (VTcca) y la retención de carbono (R).

Table 3. Estimate spacing (CAH) for optimization of combined production of sawtimber (VTcca) and carbon retention (R).

\begin{tabular}{lccccc}
\hline \multirow{2}{*}{ Lugar } & \multicolumn{2}{c}{ Relación con la cantidad de árboles por hectárea } \\
& \multicolumn{2}{c}{ VTcca $\left(\mathrm{m}^{3}\right)$} & $\begin{array}{c}\text { Cantidad de } \\
\text { árboles por } \\
\text { hectárea }\end{array}$ & $\begin{array}{c}\text { Área vital } \\
\left(\mathrm{m}^{2}\right)\end{array}$ & $\begin{array}{c}\text { Espaciamiento } \\
(\mathrm{m})\end{array}$ \\
\cline { 2 - 5 } Los Puriales & $\mathrm{VTcca}=1,4455-0,0001 \mathrm{CAH}$ & $\mathrm{R}=0,1078+0,0012 \mathrm{CAH}$ & 1.029 & 9,72 & $3,12 \times 3,12$ \\
La Felicidad & $\mathrm{VTcca}=1,7279-0,0003 \mathrm{CAH}$ & $\mathrm{R}=0,3178+0,0011 \mathrm{CAH}$ & 1.007 & 9,93 & $3,15 \times 3,15$ \\
Tres Palmas & $\mathrm{VTcca}=1,7134-0,0003 \mathrm{CAH}$ & $\mathrm{R}=0,2526+0,0012 \mathrm{CAH}$ & 974 & 10,27 & $3,20 \times 3,20$ \\
La Chispa & $\mathrm{VTcca}=2,0853-0,0009 \mathrm{CAH}$ & $\mathrm{R}=0,6772+0,0005 \mathrm{CAH}$ & 1.006 & 9,94 & $3,15 \times 3,15$ \\
Todos & $\mathrm{VTcca}=1,6133-0,0003 \mathrm{CAH}$ & $\mathrm{R}=0,2264+0,0011 \mathrm{CAH}$ & 991 & 10,09 & $3,18 \times 3,18$ \\
\hline
\end{tabular}

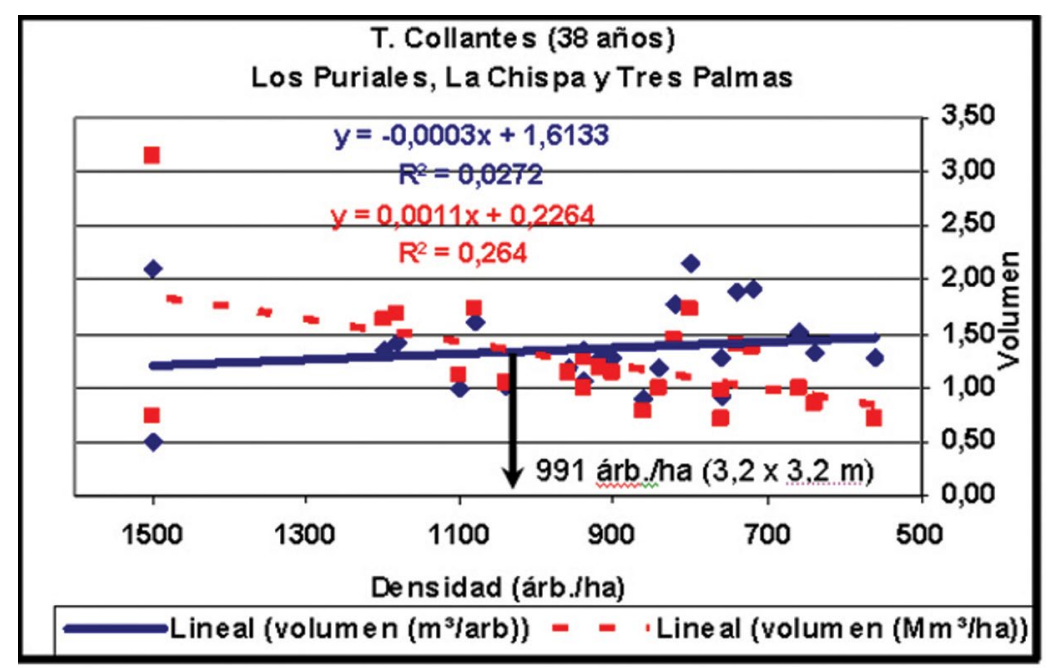

Figura 4. Estimación del espaciamiento de optimización para la combinación de la producción de madera para aserrío y la retención de carbono, en las condiciones de T. Collantes, en parcelas de $P$. caribaea con 38 años de edad.

Figure 4. Estimate spacing of optimization for combination of sawtimber production and carbon retention in T. Collantes conditions, in plots of $P$. caribaea 38 years old. 
Investigaciones sobre el efecto del espaciamiento en las variables dasométricas de las diferentes especies de pinos cubanos han sido realizadas anteriormente en el país por diversos autores, incluyendo P. caribaea M. var. caribaea B. \& G.

Acosta (1976), evaluando plantaciones de esta misma especie pero en las condiciones edafoclimáticas de Viñales, Pinar del Río, sobre suelo rojo montañoso esquelético donde el contenido de arcilla es siempre mayor en el subsuelo que en el suelo superficial, muy ácidos, pobres en materia orgánica, fósforo y potasio, aunque el contenido de calcio y magnesio es adecuado para suplir las necesidades nutricionales de los pinos, reportó que a los cuatro años y medio de la plantación no se registraban diferencias estadísticamente importantes entre el espaciamiento de $3 \mathrm{~m} \times 1 \mathrm{~m}$ y el de $3 \mathrm{~m} \times 3 \mathrm{~m}$, aunque valorando técnico-económicamente los resultados sugería utilizar los de $3 \mathrm{~m} \times 2 \mathrm{~m}$ ó de $3 \mathrm{~m} \times 2,5 \mathrm{~m}$. Sin embargo, al evaluar el mismo experimento 10 años después, Blanco et al. (1989) encontraron que todos las variables altura total, diámetro normal, volumen por árbol y poda natural, presentaban diferencias significativas entre los tratamientos; que el espaciamiento $3 \mathrm{~m}$ x 2,5 m alcanzaba los mejores resultados para las variables altura $(11,79 \mathrm{~m})$ y diámetro $(16,2 \mathrm{~cm})$, en tanto que los espaciamientos $3 \mathrm{~m} \times 1,5 \mathrm{~m}$ y $3 \mathrm{~m}$ x 2,5 m obtenían los mayores rendimientos, que fueron de 164,9 y $153,8 \mathrm{~m}^{3} /$ ha, respectivamente.

Por otra parte, Orquín et al. (1985) evaluaron la influencia del espaciamiento en el comportamiento del $\mathrm{d}_{1,30}$, la altura total y el volumen total con corteza de P. caribaea M. var. caribaea B. \& G. en T. de Collantes, considerando densidades de 2.500 (2 $\mathrm{m} \times 2 \mathrm{~m}), 1.111$ (3 $\mathrm{m} \times 3 \mathrm{~m}), 952$ (3 $\mathrm{m} \times 3,5 \mathrm{~m})$, $833(4 \mathrm{~m} \mathrm{x} 3 \mathrm{~m})$ y $500(5 \mathrm{~m} \times 4 \mathrm{~m})$ árboles por hectárea, a lo que añadieron muestras de 30 árboles en áreas con 625 (4 m x $4 \mathrm{~m})$ y $333(5,8 \mathrm{~m}$ x 5,8 m) árboles por hectárea. El detallado estudio realizado por estos autores concluyó sugiriendo el uso de diferentes espaciamientos por edades, en plantaciones destinadas a la producción de madera para aserrío en aquellas condiciones. Montalvo et al. (2007) valoraron en plantaciones de P. caribaea M. var. caribaea B. \& G. establecidas en San Pedro de Mayabón, Matanzas, sobre suelo Fersialítico Pardo Rojizo con $1.450 \mathrm{~mm}$ de precipitación anual, el efecto de diversos espaciamientos (desde $2 \mathrm{~m} \times 2 \mathrm{~m}$ hasta $4 \mathrm{~m} \times 3 \mathrm{~m}$ ) sobre la altura dominante, utilizando nueve funciones de crecimiento y rendimiento, concluyendo que en esas condiciones la altura dominante de la especie no estaba influida por el espaciamiento de la plantación.

Todos estos resultados indican que, aun cuando en diversas ocasiones ha sido analizada la influencia del espaciamiento sobre la producción de madera para aserrío en los pinos cubanos, este trabajo aborda por primera vez la combinación de este objetivo productivo con un objetivo ambiental, como es la retención de carbono, razón que origina algunas diferencias con los resultados anteriormente reportados.

\section{CONCLUSIONES}

En los rodales de P. caribaea M. var. caribaea B. \& G. establecidos en el macizo montañoso de Guamuhaya, destinados a la producción de madera para aserrío y a la retención de carbono en su biomasa, los raleos (aclareos) que se realicen no deben reducir sus existencias más allá de 990 árboles por hectárea.

La tendencia de variación presentada por el grosor de corteza con el aumento del espaciamiento, sugiere valorar su influencia sobre la retención total de carbono en esta especie.

\section{REFERENCIAS}

ACOSTA, R. Desarrollo de seis especies de pino con distintos espaciamientos, en suelos rojos montañosos esqueléticos de Viñales. Revista Forestal Baracoa, v. 6, n. 3-4, p. 3-12, 1976.

BARI, A.B.; FRÍAS, G.; HORSTEN, F. Los suelos de la Estación Experimental Forestal de Topes de Collantes, Las Villas. Revista Forestal Baracoa, v. 3, n. 3-4, p. 29-42, 1973.

BLANCO, J.J. et al. Comportamiento de Pinus caribaea var. caribaea en cinco espaciamientos de plantación en los suelos Ferralíticos Cuarcíticos Amarillos de Viñales, Cuba. Revista Forestal Baracoa, v. 19, n. 1, p. 43-54, 1989. 
BRAVO, J.A.; MONTALVO, J.M. Silvicultura de bosques naturales y plantaciones forestales. La Habana, Cuba: Instituto de Investigaciones Agro-Forestales, 2011. $34 \mathrm{p}$.

BURLEY, J.; EVANS, J.; YOUNGQUIST, J.A. Encyclopedia of forest sciences. Oxford: Elsevier, 2004. $2.093 \mathrm{p}$.

CARRERAS, R.; DECHAMPS, R. Anatomía de la madera de 157 especies forestales que crecen en Cuba y sus usos tecnológicos, históricos y culturales. I. El texto. Sciences Economiques, v. 9, p. 17-20, 1995.

HECHAVARRÍA, O. Contribución de la fenología a la conservación de tres especies forestales en Topes de Collantes. 2009. 117 p. Tesis (Doctorado en Ciencias Forestales) - Dpto. Forestal, Facultad Forestal-Agronomía, Universidad de Pinar del Río, Pinar del Río.

INTERNATIONAL PANEL ON CLIMATE CHANGE - IPCC. 2006 IPCC guidelines for national greenhouse gas inventories. In: EGGLESTON, H.S. et al. (Ed.). National Greenhouse Gas Inventories Programme. Hayama: Institute for Global Environmental Strategies - IGES, 2006.

MERCADET, A. et al. Coeficientes de carbono y nitrógeno en la madera y corteza de especies forestales arbóreas cubanas. 2011. Disponible en: $<$ http://bva.fao.cu/pub_doc/Reposit/cuf0337s.pdf $>$. Consulta: 30 abr 2012.

MONTALVO, J.M. et al. Influencia del espaciamiento inicial en el crecimiento y rendimiento de Pinus caribaea M. var. caribaea B. \& G. sobre la altura dominante. Revista Forestal Baracoa, v. 26, n. 2, p. 23-28, 2007.

MONTERO G. et al. Fijación de $\mathrm{CO}_{2}$ por Pinus sylvestris L. y Quercus pyrenaica Willd. en los montes "Pinar de Valsaín" y "Matas de Valsaín". Investigación Agraria: Sistemas y Recursos Forestales, v. 13, n. 2, p. 399-416, 2004.

ORQUÍN, J.E.; RODRÍGUEZ, E.; SÁNCHEZ, R. Influencia de los espaciamientos y raleos de Pinus caribaea M. var. caribaea B.\&G. en la zona de Tope de Collantes. Boletín Técnico Forestal, n. 3, p. 1-19, 1985.
RODRÍGUEZ, R. et al. Estimación de carbono almacenado en el bosque de pino-encino en la reserva de la biosfera El Cielo, Tamaulipas, México. Revista Ra Ximhai, v. 3, p. 317-327, 2009.

RUBIO, M. Distribución y características de los principales suelos del Escambray. Perspectivas para su utilización racional. 1986. 46 p. Tesis (opción al Título de Ingeniero Forestal) Universidad de Pinar del Río, Pinar del Río.

SNOWDON P. et al. Protocol for sampling tree and stand biomass. Canberra: Australian Greenhouse Office, 2001. 114 p. (National Carbon Accounting System Technical Report, n. 31, Draft-March 2001).

SOLER, M. Mil maderas. Valencia: Universidad Politécnica de Valencia, 2001. 598 p. 